with widespread port wine stain on his face, lower lip, arms, legs and trunk were noted. Brain magnetic resonance imaging revealed vascular malformations located periventricularly and adjacent to the corpus callosum. On brain magnetic resonance angiography ectatic, varicose deep veins (venous malformations) were detected. Lower extremity MRI showed hypertrophy of bones and soft tissues on the left side. As the child has capillary malformations (port wine stain), soft tissue and bone hypertrophy and vascular malformations a diagnosis of KTS was made. KTS consists of two major features; congenital vascular malformations and disturbed growth. For diagnosis presence of either capillary or venous malformations with disturbed growth of the bone or soft tissues is required. Children with the diagnosis of CP should be carefully examined for any finding suggesting a genetic disease.

\section{NEAR FATAL CASE OF AMLODIPINE POISONING IN AN INFANT}

doi:10.1136/archdischild-2012-302724.0567

SM Nimbalkar, DV Patel. Department of Pediatrics, Pramukhswami Medical College, Anand, India

Background and Aims Infants contribute about 5.25\% of total poisoning exposures in humans. We report first case of infant to have survived Amlodipine intoxication.

Methods 11 month old infant received 12.5 times the maximum therapeutic dose of amlodipine as a result of a medication error. $\mathrm{He}$ presented with vomiting, lethargy, breathlessness, muffled heart sounds and progressed to hypotensive shock within hours of admission. He received mechanical ventilation, fluid therapy with normal saline and inotropes. Peripheral pulses remained feeble and blood pressure was $70 / 40 \mathrm{~mm}$ of $\mathrm{Hg}$. High dose insulin infusion at $0.5 \mathrm{u} /$ $\mathrm{kg} / \mathrm{hr}$ with intravenous $25 \%$ dextrose and intramuscular glucagon for maintaining euglycemia was given. Continuous infusion of calcium gluconate at $0.5 \mathrm{mEq} / \mathrm{kg} / \mathrm{hr}$ was started simultaneously. To manage prerenal failure, oliguria and congestive cardiac failure induced pulmonary oedema peritoneal dialysis was initiated.

Result Infant improved rapidly after insulin and dextrose infusions (for 15 hours) along with glucagon and calcium gluconate infusion (for 72 hours) was initiated. Calcium channel blockers (CCB) are phenylalkyalamines. CCB act by binding to the $\alpha$ unit of the L Type calcium channels. Insulin secretion being calcium dependent, blockage of the $\mathrm{L}$ type channel results in impaired insulin secretion causing hypeglycemia. CCB poisoning results in insulin resistance. A continuous increase in blood sugar levels may predict sudden decline in hemodynamic variables rather blood pressure and pulse measurements.

Conclusion Hyperinsulinaemia/euglycaemia therapy is beneficial in seriously intoxicated patients with CCB induced hypotension, hyperglycemia, and acidosis. There may be benefit in using them in combination with other standard therapies.

\section{NEONATAL HEMOLYTIC UREMIC SYNDROME}

doi:10.1136/archdischild-2012-302724.0568

L Tiwari, N Vashisht, P Kaur, N Gupta, B Saikia, J Puliyel. Paediatrics and Neonatology, St. Stephens Hospital, Delhi, Delhi, India

Haemolytic uremic syndrome (HUS) is characterised by the triad of non immune haemolytic anaemia, thrombocytopenia and renal failure. It is rare in newborns. Only 5 cases of neonatal HUS have been reported to-date. We describe a series of 2 cases of neonatal HUS where one had definite history of birth asphyxia and another was an abandoned baby without clinical details about perinatal period. The known modalities of treatment include transfusion of plasma and plasmapheresis. In first baby haematological and renal parameters improved after double volume exchange transfusion while second baby responded to transfusion of fresh whole blood (packed red cells + plasma). Exchange transfusion essentially provides plasma exchange besides augmenting the haemoglobin levels. Arguably this is the first report of neonatal HUS treated with exchange transfusion.

\section{THORACOSCOPIC SYMPATHECTOMY FOR LONG-OT SYNDROME IN A SICKLE CELL ANEMIA PATIENT}

doi:10.1136/archdischild-2012-302724.0569

'B Banieghbal, ${ }^{2} \mathrm{~J}$ Harrisberg. 'Pediatric Surgery, Netcare Sunninghill Hospital; ${ }^{2}$ Pediatric Cardiology, Walter Sisulu Cardiac Centre for Africa, Netcare Sunninghill Hospital, Johannesburg, South Africa

Background and Aim Long QT syndrome (LQTS) is a relatively common disease, however, it is rarely described in Black African patients. We would like to present a west African patient with sickle cell disease and LQTS who was treated with thoracoscopic left sympathectomy and $1 / 3$ lower stellectomy.

Methods An 11-year old boy with sickle cell anemia ( $\mathrm{Hb}=7.2 \mathrm{~g} / \mathrm{dl})$ was also found to have LOTS. He has had $>15$ defibrillations in 5 years. He was on high dose beta-blockers but required a number of shocks despite it. Implantable defibrillator was rule out due to his home country lack of cardiac surgical expertise in case of complications. He was considered to be a suitable candidate for sympathectomy. This was done through a 3 trocar approach to the left upper chest. Sympathetic chain including stellate gangion was exposed. $20 \mathrm{mls}$ of $0.5 \%$ lignoaine was sprayed over the ganglions. Prior to excision of sympathetic chain, all visible nerves traveling medially (to the heart) was excised with hook diathermy. Lower $1 / 3$ of stellate ganglion as well as T2-T4 ganglia was resected en-bloc and submitted for histology.

Results There were no per- or post-operative complications and no evidence of Horner's syndrome post surgery. The patient was discharged 72 hours later. QTc interval before surgery was $532 \mathrm{~ms}$ and reduced to $448 \mathrm{~ms}$ prior to discharge.

Conclusion Sympathectomy including lower portion of stellate ganglion is a viable alternative for patient with LOTS

\section{PULMONARY HYPERTENSION AS A COMPLICATION OF VON RECKLINGHAUSEN DISEASE}

doi:10.1136/archdischild-2012-302724.0570

LT Celik, K Papakostas, A Artmann, TP Le. Pediatric Cardiology, Klinikum Links der Weser, Bremen, Germany

Introduction Neurofibramatosis (NF) is occasionally complicated by systemic vasculopathy such as myocardial infarction, renovascular hypertension etc. The appearance of pulmonary embolism (PE) is not desribed until yet.

Case Report 15-year-old female patient with neurofibromatosis type I. Progressive dyspnoe, general fatigue and chest pain biginning 6 days before hospital admission. Anamnestic no clinical sign for deep vein thrombosis. No oral contraceptive, non smoker. Laboratory findings: Elevated troponine, plasma brain natriuretic petide (BNP) and D-dimer. Normal ECG, echocardiogram showed right ventricular and atrial enlargement with moderate tricuspid regurgitation and estimated right ventricular systolic pressure at $45 \mathrm{~mm}$ $\mathrm{Hg}$ and dilated main pulmonary artery. Left ventricular function was normal.

Chest $\mathrm{x}$ ray showed right heart enlargement but normal lung structure. Lung CT revealed massive PE in left and right branches and a mediastinal tumor. Lysis with Alteplase was given for 4 days. Normalisation of laboratory and echocardiogram. Because of the tumor angiographic-MRI was done for follow-up and showed normal flow pattern in pulmonal vasculature. The tumor was classified as a ganglioneurome. Genetic thrombophilia testing was negative, 